\title{
Biodegradation of aromatic hydrocarbons in a compost biofilter
}

\author{
Ayoob Torkian, ${ }^{1 *}$ Reza Dehghanzadeh ${ }^{2}$ and M Hakimjavadi ${ }^{1}$ \\ ${ }^{1}$ Institute of Water \& Energy, Sharif University of Technology, PO Box 11365-688 Tehran, Iran \\ ${ }^{2}$ Department of Environmental Health Engineering, Tabriz University of Medical Sciences, Tabriz, Iran
}

\begin{abstract}
Two laboratory-scale biofilters filled with the same type of packing material were operated at different gas flow rates and influent concentrations of toluene and xylene in order to investigate their performance in treating waste gas streams. The columns contained a mixture of municipal compost as a base material and wood chips as a bulking agent in an 80:20 ratio; the porosity was $54 \%$. Microbial acclimation was achieved by addition of nutrient-enriched solution along with pollutants for a week by daily mixing and natural aeration. During the start-up of the systems with inlet concentrations of 20 and $70 \mathrm{ppm}$ for toluene and xylene, respectively, high biomass growth resulted in pressure drops in excess of $2000 \mathrm{~Pa} \mathrm{~m}^{-1}$. Under steady state conditions, the response of each biofilter to variations in contaminant mass loading was studied by either changing the influent concentration or flow rate of the inlet waste stream. The results show that organic loading rates of up to 110 and $150 \mathrm{~g} \mathrm{~m}^{-3} \mathrm{~h}^{-1}$ can be handled without any indication of the elimination capacity being saturated. However, maintaining the pressure drop below $1000 \mathrm{~Pa} \mathrm{~m}^{-1}$ to avoid operational problems, optimal organic loading rates for toluene and xylene of $78 \pm 8$ and $80 \pm 14 \mathrm{~g} \mathrm{~m}^{-3} \mathrm{~h}^{-1}$ respectively are suggested for an HRT value of $60 \mathrm{~s}$. Under these conditions, elimination capacities of $73 \pm 4$ and $73 \pm 14 \mathrm{~g} \mathrm{~m}^{-3} \mathrm{~h}^{-1}$ and removal efficiencies of $94 \pm 6 \%$ and $91 \pm 8 \%$ were achieved for toluene and xylene, respectively.

(C) 2003 Society of Chemical Industry
\end{abstract}

Keywords: air pollution; biofiltration; biofilter; volatile organic compounds (VOC); BTEX; compost; methanol; toluene

\section{INTRODUCTION}

A large quantity of volatile organic compounds (VOCs) is released from various industrial sources such as the petrochemical and printing industries, coating facilities, and hazardous wastes sites. Waste gas streams from these sources are often characterized by high flow rates and low pollutant concentrations of less than $2000 \mathrm{ppm}$. A number of physiochemical processes including adsorption, incineration, and absorption can be used to treat VOCs but the costs for chemicals and fuels as well as further treatment or disposal of secondary wastes are increasingly inhibiting adoption of these solutions. As the concern over VOCs grows, more effective and economically viable solutions will be sought.

Biofiltration has proven to be an effective and economical technology compared with other air pollution control (APC) techniques in reducing VOC and odorous emissions. ${ }^{1}$ It is considered to be a clean technology, with minimal energy requirements and low waste production. The process involves contacting contaminated streams with microbial biofilms immobilized on porous support particles. The pollutants diffuse from the gas phase into the thin layer of biofilm attached to the support media and are metabolized. The end products of the complete biodegradation are $\mathrm{CO}_{2}$, water and microbial biomass. ${ }^{2}$

Compost-based media have been used extensively in recent years because they are cheap and have diversified microbial communities capable of degrading various pollutants once they become acclimatized to the substrate. Various bulking materials have been used by many researchers, including porous clay, polystyrene spheres, perlite pellets, wood chips and polyurethane foam, to reduce bed pressure drop..$^{3,4}$

In recent years a large number of studies have been conducted on the biofiltration of contaminated gas streams. The contaminants include various hydrophobic and hydrophilic compounds found in different industries. Within the hydrophobic category, benzene, toluene, ethylbenzene and xylene (BTEX) compounds, and within the hydrophobic category methyl and ethyl-containing substances, have been more extensively studied. Strauss $e t a l^{5}$ studied the toluene removal efficiency of a composted pine bark biofilter. Removal efficiencies exceeding $90 \%$ were obtained when the biofilter was subjected to a gas

* Correspondence to: Ayoob Torkian, Institute of Water \& Energy Sharif University of Technology, PO Box 11365-688 Tehran, Iran

E-mail: torkian@sharif.edu

(Received 1 March 2003; revised version received 8 October 2002; accepted 12 January 2003) 
retention time in excess of $0.32 \mathrm{~min}$ and loading rates below $42 \mathrm{~g} \mathrm{~m}^{-3} \mathrm{~h}^{-1}$. Ortiz et $a l^{6}$ evaluated the ability of organic and inorganic supports to remove volatile organic compounds from gasoline vapors in $4-\mathrm{dm}^{3}$ bench-scale reactors inoculated with an adapted consortium. The supports were peat, a mixture of vermiculite and activated carbon (V-AC) barks, and porous $15 \mathrm{~mm}$ glass Raschig rings. A mixture of benzene, toluene and xylene (BTX) at $200 \mathrm{gC} \mathrm{m}^{-3} \mathrm{~h}^{-1}$ was tested for more than 100 days the four biofilters with an empty bed retention time (EBRT) of $60 \mathrm{~s}$. Removal efficiencies higher than $95 \%$ were obtained with V-AC, $85 \%$ with barks, and $65 \%$ with the Raschig rings. In all cases, drying problems were observed into beds after several days of operation. In the other research three laboratory-scale biofilter columns containing a mixture of yard waste and sludge compost as the base biofilter material were examined for the removal of BTEX. In this study, removal efficiencies of greater than equivalent to $90 \%$ were achieved for inlet concentrations of greater than equivalent to $200 \mathrm{ppmv}$ of each of the BTEX compounds at a gas loading rate of $17.6 \mathrm{~g} \mathrm{~m}^{-3} \mathrm{~h}^{-1}$ in all columns over the period of study. Matteau and Ramsay ${ }^{7}$ studied the biofiltration of toluene with compost consisting of leaves and alfalfa. Under mesophilic conditions, the maximum biodegradation rates were near $89 \mathrm{~g} \mathrm{~m}^{-3} \mathrm{~h}^{-1}$ at an inlet concentration of about $5 \mathrm{~g} \mathrm{~m}^{-3}$ and a gas residence time of $90 \mathrm{~s}$.

This paper reports the results of a bench-scale study to examine the response of biofilter systems to variations of inlet concentration, moisture content, and loading (organic and hydraulic) conditions. Specifically, the effects of inlet concentration and organic loading rate on the performance of the biofilter in treating toluene and xylene were studied.

\section{MATERIALS AND METHODS}

\subsection{Experimental set-up}

Two three-stage biofilters constructed from galvanized iron were used in this study (Fig 1). The columns had an inner diameter of $8 \mathrm{~cm}$ and an effective height of $150 \mathrm{~cm}$. Perforated steel plate plenums (pore diameter $=2 \mathrm{~mm}$ ) placed between sections acted as a support for the packing material as well as for flow redistribution. A $30 \mathrm{~mm}$ space in between the sections allowed for representative gas sampling. Provision of sampling ports at the midpoint within each section allowed temperature measurements as well as access to the bed media.

The synthetic waste air stream was prepared by sending compressed air from a house distribution system through a granular activated carbon canister to adsorb residual oil. Gas flow was controlled using pressure regulators and flow meters. The air stream was then sparged through a $5 \mathrm{dm}^{3}$ container equipped with a heated element for temperature control. Pollutant vapor was prepared by injecting a low-flow air stream into a liquid pollutant stock

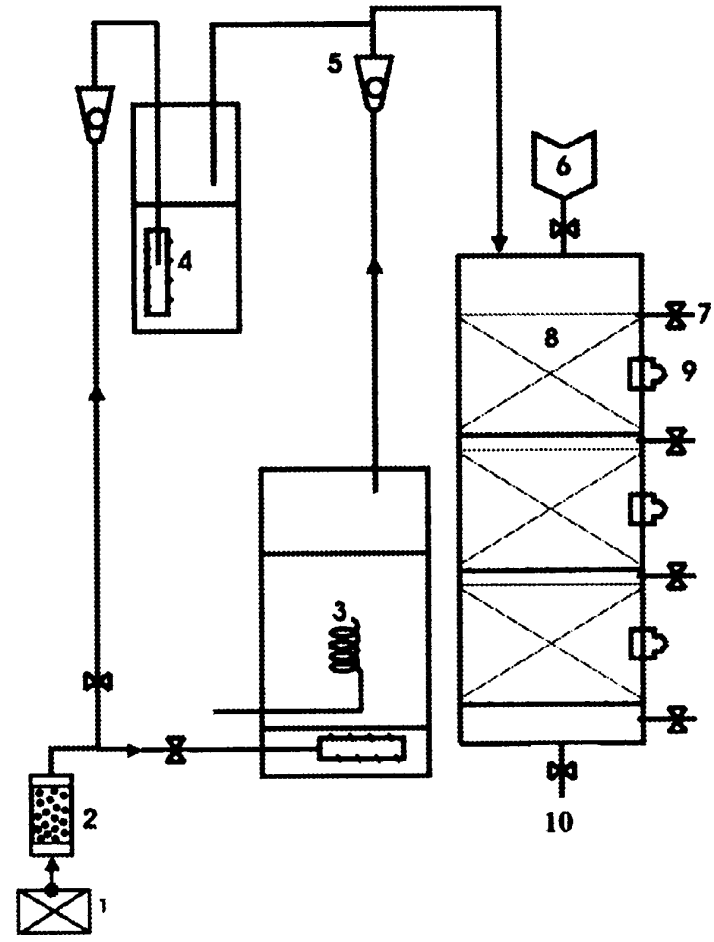

Figure 1. Schematic of biofilter system (1-compressor, 2 - carbon filter, 3-humidifier, 4 - pollutant vessel, 5-rotameter, 6 - nutrient, 7 - gas sampling port, 8 - biofilter bed, 9 - bed sampling port, 10 - leachate).

solution. After humidification, the main air stream was mixed with the stream containing pollutant vapor to generate feed air with the required concentration. Variation of humidity in the influent gas stream and biofilter material was controlled by changing the water temperature in the humidifier. Temperature control of the bed material was achieved by using a heated tape wrapped around the exterior of the reactor wall. During the steady state operational period of the study, bed temperature and humidity were maintained at $30 \pm 1{ }^{\circ} \mathrm{C}$ and $60-65 \%$, respectively.

\subsection{Compost media}

Municipal compost was obtained from a local composting facility (Tehran Compost Co) and filtered to remove visible dirt and large floc particles. The chemical composition of the bed compost is shown in Table 1 . Wood chips $(0.5-2 \mathrm{~cm})$ were added as a bulking material to produce an $80: 20 \mathrm{w} / \mathrm{w}$ ratio of compost-wood chip. In preparing the packing medium, wood chips were screened and placed in water for a few hours to-enhance the attachment of compost to the chips. The microbial population

Table 1. Chemical composition of the compost

\begin{tabular}{lc}
\hline Characteristic & $\%(\mathrm{w} / \mathrm{w})$ \\
\hline Moisture & 32 \\
Total organic carbon & 25 \\
Nitrogen $(\mathrm{N})$ & 1.7 \\
Phosphorus $\left(\mathrm{P}_{2} \mathrm{O}_{5}\right)$ & 0.46 \\
Potassium $\left(\mathrm{K}_{2} \mathrm{O}\right)$ & 0.85 \\
\hline
\end{tabular}


was acclimatized to the environment for about 7 days prior to the start up by adding the following nutrient and buffering solution (units in $\mathrm{g} \mathrm{dm}^{-3}$ ): $\mathrm{KH}_{2} \mathrm{PO}_{4}, 0.54 ; \mathrm{K}_{2} \mathrm{HPO}_{4}, 0.54 ; \mathrm{NH}_{4} \mathrm{NO}_{3}, 0.5 ; \mathrm{NaCl}$, $0.26 ; \mathrm{CaCl}_{2} \cdot 2 \mathrm{H}_{2} \mathrm{O}, 0.025 .^{8} \mathrm{~A} 1 \mathrm{dm}^{3}$ trace element solution was also added to the bed, resulting in an overall $\mathrm{C} / \mathrm{N}$ ratio of around 30 .

\subsection{Operational and performance parameters}

Operational and performance parameters include loading rate, elimination capacity, and detention time. Loading rate can be looked at from the pollutant, waste gas flow rate, bed volume and/or surface, and microbial mass. Mass loading rate $\left(L_{\mathrm{m}}\right)$ takes into account the gas flow rate and contaminant concentration and is defined as the mass of pollutant introduced in a unit volume of biofilter material per unit time (eg g toluene per $\mathrm{m}^{3}$ media per hour). As such, this parameter integrates media characteristics and biofilm mass and activity into the volume of media.

Elimination capacity $(E C)$, a performance indicator, is related to mass loading rate in that it is defined as the fraction of the mass loading rate biodegraded in the biofilter. It differs from removal efficiency $(\eta)$, an operational parameter, which is a measure of the effectiveness of the biofilter in degrading a contaminant. Elimination capacity is a useful parameter for design purposes and removal efficiency helps the operator determine if the system is complying with regulatory requirements.

Surface loading rate $\left(L_{\mathrm{s}}\right)$ is a measure of the volume of waste gas 'seen' by the area of active media perpendicular to the flow stream per unit time similar to the air-to-cloth ratio in fabric filter air pollution control technology $\left(\mathrm{eg} \mathrm{m}^{3} \mathrm{~m}^{-2} \mathrm{~h}^{-1}\right.$ or superficial or face velocity, $\mathrm{m} \mathrm{h}^{-1}$ ). This is different from the interstitial velocity, which determines the column pressure drop and is defined as the volume of the waste gas per unit area of the openings within the biofilter material per unit time. The latter is more difficult to estimate due to the transient nature of the void fraction and permeability of the column, which is in turn a function of biofilm growth and media compaction.

Mass loading rate $\left(\mathrm{g} \mathrm{m}^{-3} \mathrm{~h}^{-1}\right)$ and elimination capacity $\left(\mathrm{g} \mathrm{m}^{-3} \mathrm{~h}^{-1}\right)$ were determined using the relationships between influent and effluent contaminant concentration, waste air flow rate, and the effective volume of biofilter column as follows:

$$
\begin{aligned}
& L_{\mathrm{m}}=\left[\frac{Q}{V}\right] C_{\text {in }}\left[\frac{M \times 10^{-3}}{25.3}\right] \\
& E C=\left[\frac{Q}{V}\right]\left(C_{\text {in }}-C_{\text {out }}\right)\left[\frac{M \times 10^{-3}}{25.3}\right]
\end{aligned}
$$

where $Q$ is the waste gas flow rate $\left(\mathrm{m}^{3} \mathrm{~h}^{-1}\right), V$ is the volume of filter bed $\left(\mathrm{m}^{3}\right), M$ is the molecular weight of the contaminant, and $C_{\text {in }}$ and $C_{\text {out }}$ are the contaminant concentrations in the influent and effluent waste gas stream (ppmv).

\subsection{Analytical methods}

\subsubsection{Gas concentration}

In order to evaluate biofilter performance, gas samples were collected at the inlet, outlet, and in the $30 \mathrm{~mm}$ plenum between the sections. Gas samples were collected in $5-\mathrm{dm}^{3}$ Tedlar bags by connecting the bag port onto the tube connected to the biofilter sampling ports. The Tedlar bags were flushed with activated carbon-filtered air prior to sampling. Analysis was done within $3 \mathrm{~h}$ using a gas chromatograph (Shimadzu Model 14A) equipped with a flame ionization detector and with a $3 \mathrm{~m}$ packed column (SV-30 Shimadzu). The temperature schedule was: injector $200^{\circ} \mathrm{C}$, oven $200^{\circ} \mathrm{C}$, detector $250^{\circ} \mathrm{C}$ and nitrogen was used as carrier gas $\left(50 \mathrm{~cm}^{3} \mathrm{~min}^{-1} \mathrm{~cm}^{3}\right)$. Gas samples of $1 \mathrm{~cm}^{3}$ containing the target contaminants were obtained from Tedlar bags using a $2 \mathrm{~cm}^{3}$ gas-tight syringe (VICI Inc) and were injected immediately into the GC unit for concentration determination. The inlet and outlet concentrations reported represent an average of two sequential samples. The detection limit of the toluene and xylene gas analyses was $10 \mathrm{ppmv}$. Non-detect samples were set to zero for all calculations, including steady-state analyses.

Unknowns were determined from a calibration curve prepared from peaks observed for known concentrations of toluene and xylene. The standards were prepared by introducing known volumes of toluene and xylene into the $5 \mathrm{dm}^{3}$ sealed Tedlar bag. The above volumes were selected based on vapor pressure calculations at room temperature and pressure. The equilibrium vapor pressures were calculated to be 2.92 and $12.73 \mathrm{kPa}$ (288 and $1256 \mathrm{ppm})$ at $20^{\circ} \mathrm{C}$ and $101.325 \mathrm{kPa}$ for toluene and xylene using Antoine's equation. Considering the volume of the Tedlar bag and maximum concentrations possible at equilibrium, known liquid volumes were injected. For example, for a $5 \mathrm{dm}^{3}$ Tedlar bag, $2.2 \mathrm{~mm}^{3}$ toluene should be injected to achieve a concentration of $100 \mathrm{ppm}$. To ensure sufficient toluene reserve was available, $5 \mathrm{~mm}^{3}$ toluene was introduced for this concentration. At least $3 \mathrm{~h}$ was allowed prior to sample withdrawal for GC injection and analysis to ensure sufficient time was available to reach liquid-vapor equilibrium conditions.

\subsection{2 pH measurement}

For $\mathrm{pH}$ measurement $5 \mathrm{~g}$ of bed medium was mixed with $1000 \mathrm{~cm}^{3}$ of distilled water.

\subsubsection{Moisture content}

Bed samples were taken through a special port connected to a spoon-like holder by removing $15 \mathrm{~g}$ of medium each time. The mass thus removed constituted only a small fraction of bed mass so no replacement of the medium was done to compensate for mass reduction resulting from sampling. Media make-up was only done at the beginning and end of a given experimental run. Moisture content was determined by drying $10 \mathrm{~g}$ of the bed medium at 
$104^{\circ} \mathrm{C}$ for $24 \mathrm{~h}$, cooling in desiccators to room temperature, and measuring the final weight. ${ }^{9}$

\subsubsection{Temperature}

Heated tape was wrapped around the bed exterior and connected to a precision thermostat (Aibin Co) to control temperature within $1^{\circ} \mathrm{C}$. The bed was further insulated with glass wool to reduce heat exchange with the ambient environment. Temperature was maintained at $30^{\circ} \mathrm{C}$ and measured using alcohol in a glass thermometer with a range from -10 to 110 and a scale division of $1^{\circ} \mathrm{C}$.

\subsubsection{Gas flow rate}

Gas flow rates were measured using flow meters (Omega FI-2016) with units of $\mathrm{dm}^{3} \mathrm{~min}^{-1}$.

\subsubsection{Pressure drop}

A water-filled manometer with a minimum division length reading of $1-\mathrm{mm}$ water column was used to measure pressure drop across the column.

\section{RESULTS AND DISCUSSION}

\subsection{Start-up}

The biofilters were acclimatized by feeding toluene and xylene at inlet concentrations of 50 and $20 \mathrm{ppm}$ and organic loading rates of 17 and $4 \mathrm{~g} \mathrm{~m}^{-3} \mathrm{~h}^{-1}$, respectively. Humidity was maintained in the $60-70 \%$ range, and hydraulic residence time (HRT) was kept constant at $60 \mathrm{~s}$. The results are shown in Fig 2.

Inlet concentration is one of the parameters influencing biofilter performance and is usually normalized as loading to unit bed volume or surface. Higher pollutant concentrations result in increased mass transfer rates from the gas phase to the biofilm medium. It also makes sufficient substrate accessible for microbial growth. However, excessive concentrations of refractory compounds with low biodegradability can adversely affect the metabolic activities of the microbial population. ${ }^{10}$ As such, a gradual increase during the initial phases of reactor operation is essential for proper microbial growth and development.

As shown in Fig 2, there is an increasing trend of removal efficiency with increased pollutant concentration fed into the biofilter, reaching $90 \%$ for toluene and close to $100 \%$ for xylene in less than 5 days. The observed acclimatization period in this study was shorter than the $2-3$ weeks reported in the literature for toluene and xylene. ${ }^{11}$ The reason for the shorter acclimatization period for our system can be attributed to the fact that prior to the start-up of the biofilters, the bed media were mixed with high concentrations of these pollutants and the necessary nutrients to increase
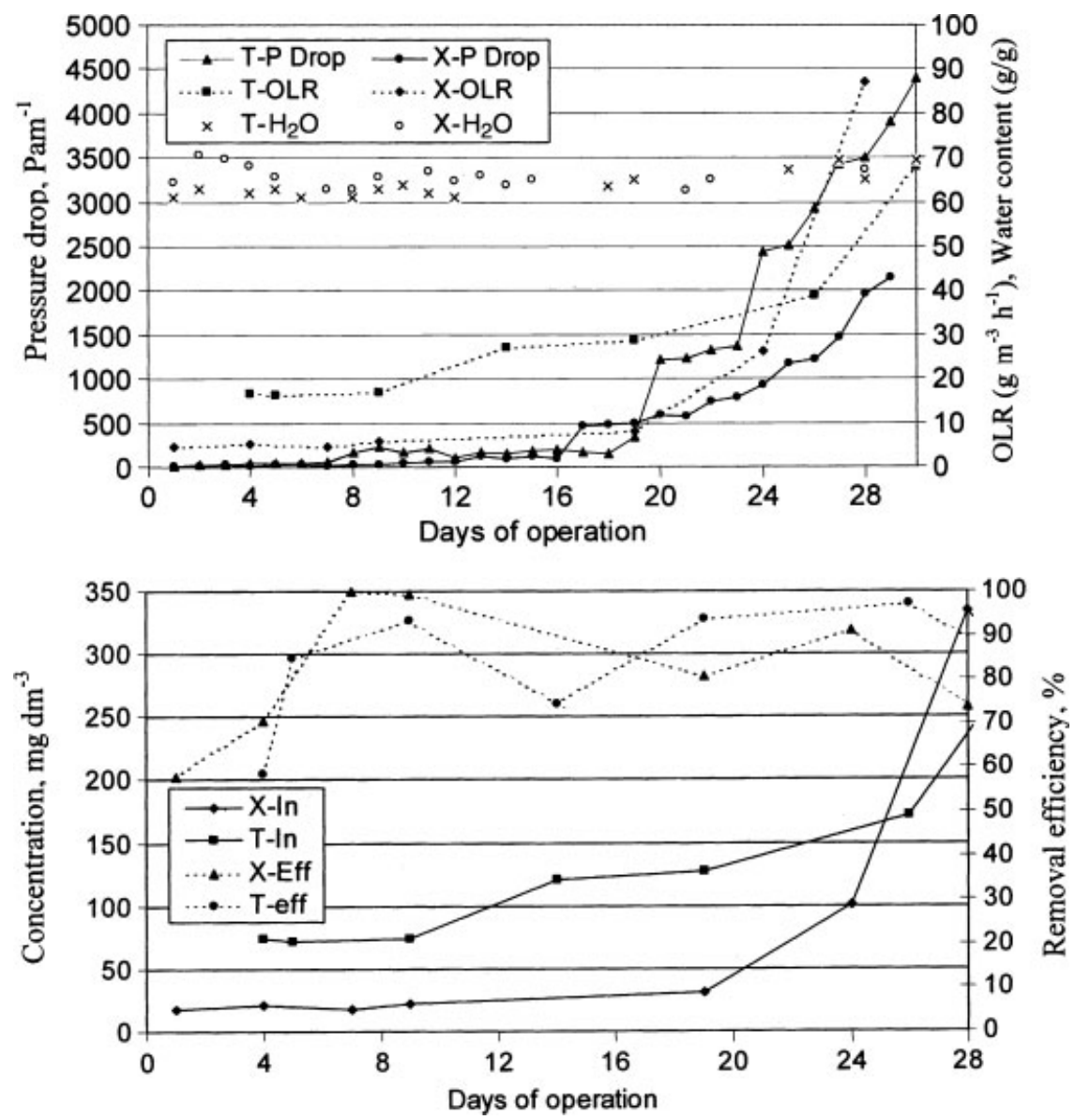

Figure 2. Variation of bed pressure drop (P drop) and removal efficiency of toluene $(T)$ and xylene $(X)$ during the start up of the biofilter (In = influent, Eff = effluent). 
the mass and number of specific microbes having the capability to degrade the pollutants.

More fluctuation of removal efficiencies was observed later in the experiments but the removal efficiencies did not fall below $90 \%$ for toluene and below $70 \%$ for xylene, indicating gradual acclimatization of microbes to the pollutants. Various factors including excessive pressure drops due to overgrowth of biofilm could have been responsible for variations, in removal efficiencies.

Pressure drop across the biofilter bed increased with increasing organic loading rates and the consequent growth of microorganisms and reduction in porosity. The highest pressure drops of 500 and $250 \mathrm{~mm} \mathrm{H}_{2} \mathrm{O}$ were observed around day 30 and the normalized values for unit height are shown in Fig 2 . Since operation of the biofilters was not feasible under these condition, the systems were shut down and the compost remixed. Remixing is known to improve operating (reduced channeling effect and bed drying or clogging, and improved particle homogenization) and environmental conditions (nutrients, water, oxygen, and redistribution of biofilm), resulting in better biofilter performance..$^{8,12}$ The rate of increase in pressure drop was reduced making a steady state operation of the biofilters feasible at a moisture content of $60-65 \%$.

\subsection{Removal efficiency}

Removal efficiency of pollutants is an important parameter influencing the degree of compliance of the control systems with emission regulations. Keeping the hydraulic residence time constant at $60 \mathrm{~s}$, the contaminant feed rate was increased stepwise so that mass loading changed proportionally and the response was investigated. The results shown in Fig 3 indicate that removal efficiencies fluctuate between 70 and $100 \%$ at different operational periods. For the range of concentrations examined, biofilter performance within the range of 50-600 ppm for toluene and $20-400 \mathrm{ppm}$ for xylene produced effluent concentrations below the $50 \mathrm{ppm}$ local regulatory standard.

At the beginning of each step change in feed concentration, there was a larger than normal fluctuation in removal efficiency, with a gradual recovery and increase in removal efficiency with time. For example, with increased toluene and xylene concentrations $300 \mathrm{ppm}$ at day 28, removal efficiency was decreased to $86 \%$ and $72 \%$, respectively. The time to recover is shorter for toluene than xylene. The same trend of decreased removal efficiency and recovery is observed at day 72 but because the increase in concentration is higher as compared with day 28 , the removal efficiency is lowered to $65 \%$ for toluene. The observed lower removal efficiencies can be tolerated for short periods of time but may result in violation of emission standards should they last for a long time. The length of this time is dependent on the operational conditions and the low near elimination capacity is to being saturated. This is investigated in the next section.

\subsection{Elimination capacity}

Increased influent pollutant concentration can result in reduced removal efficiencies, as shown in the previous section. The appearance of and continuing low removal efficiencies can be a good indicator of poor environmental and/or operational conditions for resident microbial population. In order to prevent the onset of unfavorable conditions, elimination capacity was monitored and the results are presented in Fig 4. This figure shows that there is a linear relationship between elimination capacity and organic loading rate for both toluene and xylene indicating that saturation is not being reached. The data were more scattered at higher organic loading rates but high correlation coefficients indicate no adverse impact on performance.

The slope of the linear relationship for toluene is slightly less steep than for xylene indicating a higher biodegradability for xylene but considering the statistical variability of the experiment data, this statement should be treated cautiously. In fact, the results from the initial period of experiments show otherwise. As explained before, during the start-up period, application of higher organic loading rates was possible for toluene and the time for adaptation was also shorter than that for xylene. It should be noted

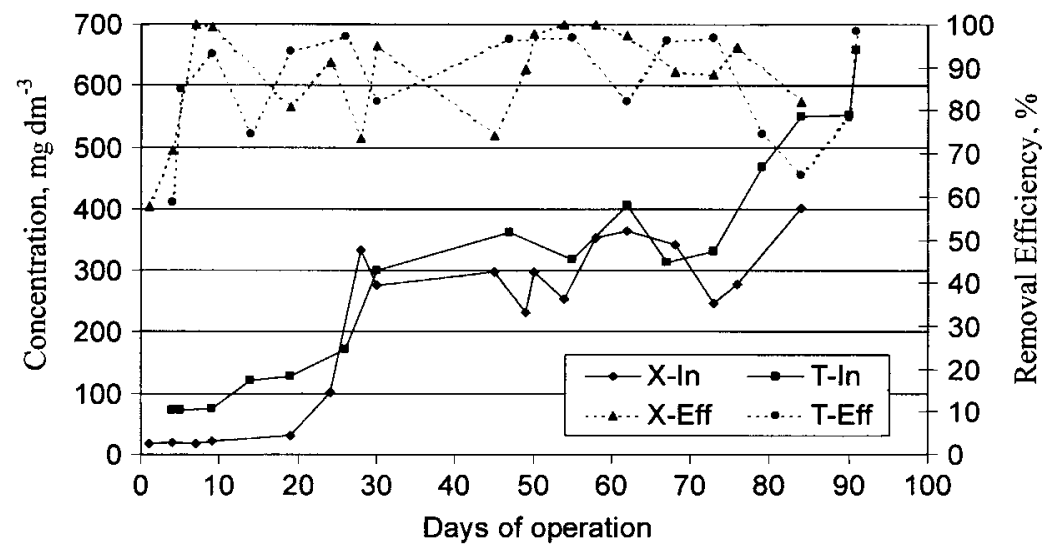

Figure 3. Variation of removal efficiency of toluene $(T)$ and $x y l e n e(X)$ at different organic loading rates (In $=$ influent, Eff $=$ effluent). 


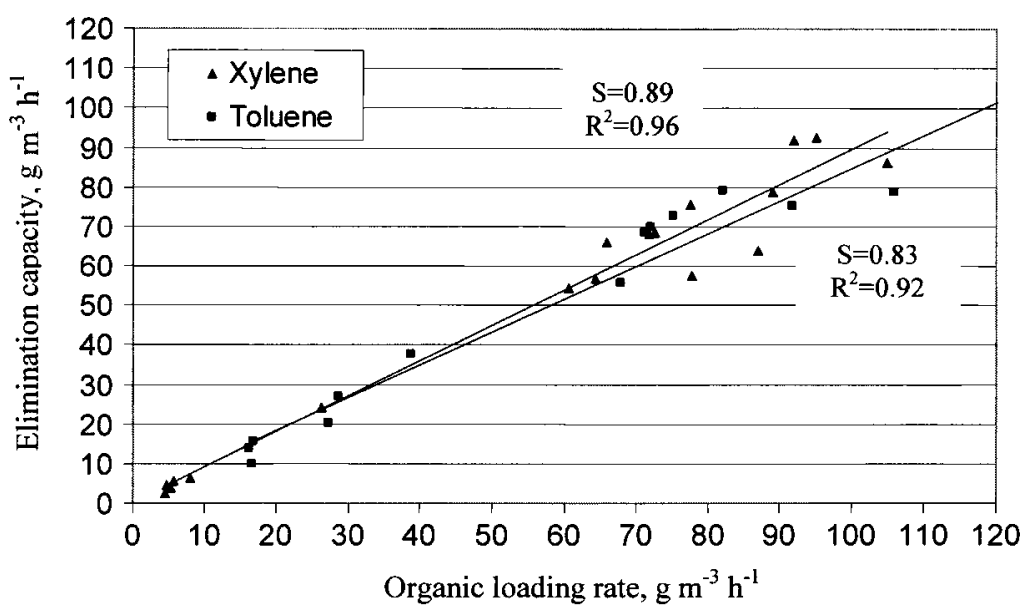

Figure 4. Elimination capacities of biofilter system at different organic loading rates.
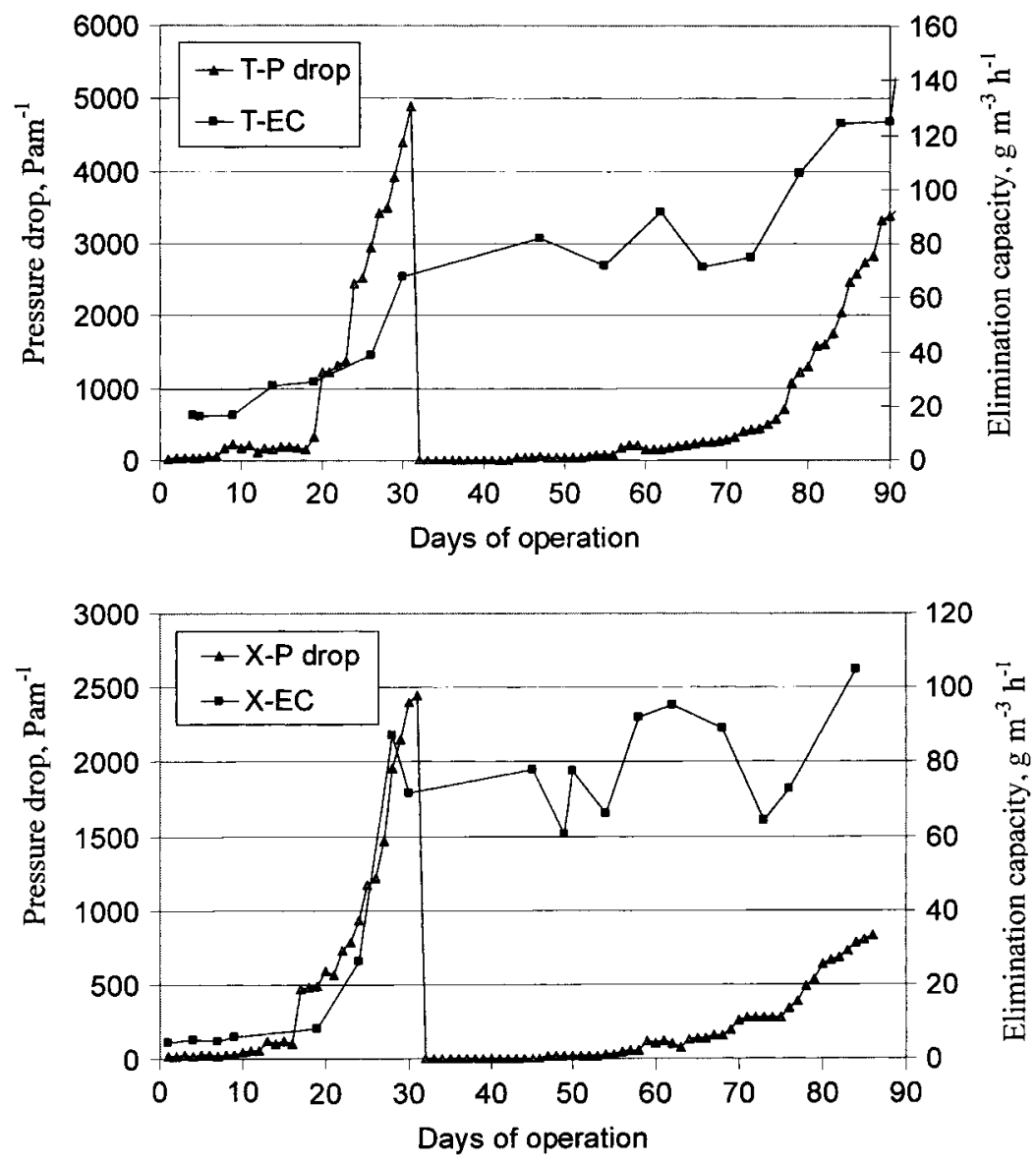

Figure 5. Variation of biofilter bed pressure drop and elimination capacity during the study.

that the findings in previous studies show a higher degradability for toluene. ${ }^{13}$

Based on these results it can be surmised that since the available data indicate no exhaustion of elimination capacity, organic loading rates as high as 110 and $150 \mathrm{~g} \mathrm{~m}^{-3} \mathrm{~h}^{-1}$ are possible for toluene and xylene, respectively. Previous studies show that removal of xylene at organic loading rates higher than $75 \mathrm{~g} \mathrm{~m}^{-3} \mathrm{~h}^{-1}$ is zero order and thus independent of inlet concentration. ${ }^{14}$ For toluene, a linear relationship between organic loading rates and elimination capacity up to $100 \mathrm{~g} \mathrm{~m}^{-3} \mathrm{~h}^{-1}$ has been reported. ${ }^{13}$

\subsection{Column pressure drop}

Resistance to flow was continuously monitored throughout the experiment using a water manometer. As shown in Fig 5, there was a gradual pressure buildup with time due to generation and accumulation of biomass and the accompanying reduction in the bed void fraction as a result of substrate oxidation reactions. The two columns have almost identical pressure drop patterns but with higher values per unit height for toluene than xylene. This difference is possibly due to greater microbial biomass growth resulting from the higher biodegradability of the 
toluene, but no actual enumeration was performed. However, this suggestion is also supported by the observation that the time to reach inhibitory pressure drops was shortened at higher flow rates (lower EBRTs) but with constant inlet pollutant concentrations. Higher flow rates means higher organic loads and thus more substrate is available for the growth of biofilm. This trend can be continued to a point where either pressure drops inhibits further operation or the point of maximum mass loading for the biomass is reached.

With time, overgrowth of microorganisms coupled with bed compaction resulting from cluster formation, shrinkage, and formation of cracks in the compost material gradually reduced the void space, resulting in increased pressure drop. Furthermore, dry patches were observed in the initial portion of the first sections of the columns due to higher biological activity and increased water evaporation due to energy being released from biooxidation. Although no direct measurement of microbial mass was performed, results shown above indicate that up to $50 \%$ of the overall removal efficiencies are related to the initial section of biofilters. No drying was observed in the deeper reaches of the bed because of the downflow operational mode of the reactors and variable densities of water and air. To alleviate these problems, the media were removed and remixed. This resulted in significant reduction in pressure drops, as expected. This observation is in line with experiences of other researchers indicating that mixing of media reduces channeling, drying or clogging and leads to a more homogeneous particle distribution, and redistribution of water, oxygen, nutrients and biofilm. 8,15

The results showed that in the first 4 weeks and the steady state period after that, increased organic loading rate does not adversely impact the elimination capacity of the biofilters but pressure drops become inhibitive. As such, a compromise was suggested to keep the pressure drop below $1000 \mathrm{~Pa} \mathrm{~m}^{-1}$. Based on this criterion, the corresponding time periods for toluene and xylene were 47,73 and $45-86$ days with organic loading rates of $78 \pm 8$ and $80 \pm 14 \mathrm{~g} \mathrm{~m}^{-3} \mathrm{~h}^{-1}$, respectively. At this pressure drop range, it may not be possible to realize the full elimination capacity of the biofilters but operational considerations dictate this limitation. At the above pressure drop range, sustainable EC values for toluene and xylene were $73 \pm 4$ and $73 \pm 14 \mathrm{~g} \mathrm{~m}^{-3} \mathrm{~h}^{-1}$, respectively.

\section{CONCLUSIONS}

Biological treatment technologies are reliable, efficient, and easy to maintain in treating large-volume low-concentration contaminated air streams. The results from this study show organic loading rates of up to 110 and $150 \mathrm{~g} \mathrm{~m}^{-3} \mathrm{~h}^{-1}$ can be applied for toluene and xylene, respectively, but excessive column pressure drops prevents these high loading rates. Keeping pressure drops below $1000 \mathrm{Pam}^{-1}$, organic loading rates of $78 \pm 8$ and $80 \pm 14 \mathrm{~g} \mathrm{~m}^{-3} \mathrm{~h}^{-1}$ could be achieved with EC values of $73 \pm 4$ and $73 \pm 14 \mathrm{~g} \mathrm{~m}^{-3} \mathrm{~h}^{-1}$ for toluene and xylene, respectively.

\section{REFERENCES}

1 Devinny JS, Deshusses MA and Webster TS, Biofiltration for air pollution control. $1^{\text {st }}$ Edition. CRC Press Inc, Boca Raton, FL, (1998).

2 Cox HI and Deshusses MA, Innovative experimental setup for the parallel operation of multiple bench scale biotrickling filters for waste air treatment. Environmental Technology 21:427-435 (2000).

3 Abumaizer RJ, Smith EH and Kocher W, Analytical model of dual-media biofilter for removal of organic air pollutants. ASCE F Environmental Engineering 123(6):606-614 (1997).

4 Abumaizer RJ, Kocher W and Smith EH, Biofiltration of BTEX contaminated air streams using compost-activated carbon filter media. F Hazardous Materials 60(2):111-126 (1998).

5 Strauss JM, Du Plessis CA and Riedle KHJ, Empirical model for biofiltration of toluene. ASCE $\mathcal{F}$ Environmental Engineering 126(7):644-648 (2000).

6 Ortiz L, Morales M, Gobbee G, Revah S, Guerrero VM and Auria R, Biofiltration of gasoline VOCs with different support media, Proceedings of the Air \& Waste Management Association's Annual Meeting \& Exhibition, Pittsburgh, PA, USA (1998).

7 Mateau Y and Ramsay B, Active compost biofiltration of toluene. Biodegradation 8(3): 135-141 (1997).

8 Auria R, Frere G, Acuna ME and Revah S, Influence of mixing and water addition of the removal rate of toluene vapors in a biofilter. Biotechnology $\mathcal{E}$ Bioengineering 68(4):438-445 (2000).

9 APHA, Standard Methods for the Examination of Water and Wastewater 17th edn. American Public Health Association, American Water Works Association, Water Pollution Control Federation, (1985).

10 Leson $G$ and Winer AM, Biofiltration: an innovative air pollution control technology for VOC emissions. I Air $\mathcal{E}$ Waste Management Association 41(8):1045-1054 (1991).

11 Morales M, Revah S and Auria R, Start-up and the effect of gaseous ammonia additions on a biofilter for the elimination of toluene vapors. Biotechnol E Bioenergy 60(4):483-491 (1998).

12 Mohseni $M$ and Allen DG, Biofiltration of mixture of hydrophilic and hydrophobic volatile organic compounds. Chemical Engineering Science 55:1545-1558 (2000).

$13 \mathrm{Wu}$ G, Conti B and Leroux A, A high performance biofilter for VOC emission control. I of the Air \& Waste Management Association 49:185-191 (1999).

14 Jorlo H, Bibeau L, Viel G and Heitz M, Effects of gas flow rate and inlet concentration of xylene vapors biofiltration performance. Chemical Engineering f 76(3):209-221 (2000).

15 Wang JZ, Summers RJ and Miltner RJ, Biofiltration performance. I. Relationship to biomass. $f$ Am Water Works Association 87(12):64-70 (1995). 